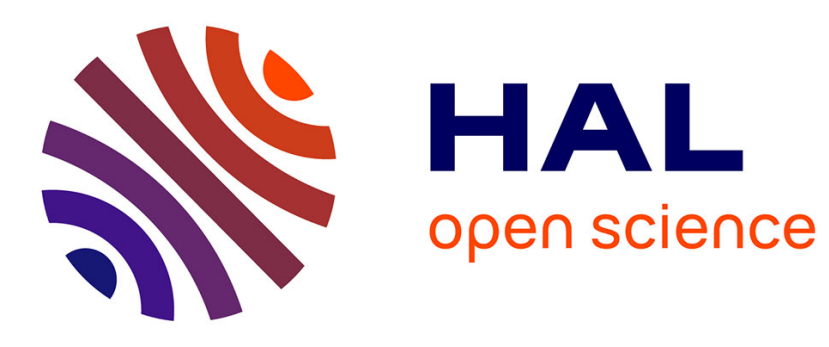

\title{
Pazopanib efficacy in recurrent central nervous system hemangiopericytomas
}

Caroline Apra, Agusti Alentorn, Karima Mokhtari, Michel Kalamarides, Marc

Sanson

\section{- To cite this version:}

Caroline Apra, Agusti Alentorn, Karima Mokhtari, Michel Kalamarides, Marc Sanson. Pazopanib efficacy in recurrent central nervous system hemangiopericytomas. Journal of Neuro-Oncology, 2018, 139 (2), pp.369-372. 10.1007/s11060-018-2870-0 . hal-01992423

\section{HAL Id: hal-01992423 \\ https: / hal.sorbonne-universite.fr/hal-01992423}

Submitted on 24 Jan 2019

HAL is a multi-disciplinary open access archive for the deposit and dissemination of scientific research documents, whether they are published or not. The documents may come from teaching and research institutions in France or abroad, or from public or private research centers.
L'archive ouverte pluridisciplinaire HAL, est destinée au dépôt et à la diffusion de documents scientifiques de niveau recherche, publiés ou non, émanant des établissements d'enseignement et de recherche français ou étrangers, des laboratoires publics ou privés. 


\title{
Pazopanib efficacy in recurrent central nervous system hemangiopericytomas
}

\author{
Caroline Apra $^{1}$ (1) $\cdot$ Agusti Alentorn $^{1,2} \cdot$ Karima Mokhtari $^{3} \cdot$ Michel Kalamarides $^{1,4} \cdot$ Marc Sanson $^{1,2}$
}

\begin{abstract}
Introduction There is currently no treatment for solitary fibrous tumors/hemangiopericytomas $(\mathrm{SFT} / \mathrm{H})$ of the central nervous system recurring after multiple surgeries and radiotherapies. The NAB2-STAT6 gene fusion is the hallmark of these tumors, and upregulates Early Growth Factor, activating several growth pathways.

Methods We treated two patients presenting pluri-recurrent meningeal SFT/H with Pazopanib, a broad-spectrum tyrosine kinase inhibitor. We analyzed the exome and RNA sequencing data of one of them and, in addition to another meningeal $\mathrm{SFT} / \mathrm{H}$, compared it to the transcriptomic profiling of 5 systemic SFT/H.

Results A dramatic clinical and radiological response was observed in both cases, respectively 84 and $43 \%$ decrease after 3 months. As a comparison, Pazopanib has only a stabilizing effect in systemic SFT/H. Indeed, central nervous system SFT/H show overexpression of different tyrosine kinases targeted by Pazopanib.

Conclusions Two consecutive patients with untreatable central nervous system SFT/H showed a spectacular partial response to Pazopanib, an unprecedented result in SFT/H. This result could be explained by differences in expression profiles and calls for a confirmation in a larger cohort of patients.
\end{abstract}

Keywords Pazopanib $\cdot$ Hemangiopericytoma $\cdot$ Solitary fibrous tumor $\cdot$ Fusion gene $\cdot$ NAB2 $\cdot$ STA6

In the new 2016 WHO classification, meningeal solitary fibrous tumors/hemangiopericytomas $(\mathrm{SFT} / \mathrm{H})$ represent a rare unique tumor entity characterized by the constant presence of the NAB2-STAT6 gene fusion. The resulting STAT6positive nuclear staining is the hallmark of the disease, also present in systemic SFT/H [1, 2]. SFT/H range from benign grade I fibrohyaline tumors, that are usually surgically

Electronic supplementary material The online version of this article (https://doi.org/10.1007/s11060-018-2870-0) contains supplementary material, which is available to authorized users.

Marc Sanson

marc.sanson@aphp.fr

1 Sorbonne Université, UMR S 1127, Inserm U 1127, CNRS UMR 7225, ICM, 75013 Paris, France

2 Department of Neurology, AP-HP, Groupe Hospitalier Pitié Salpêtrière, 75013 Paris, France

3 Laboratoire de Neuropathologie R Escourolle, AP-HP, Groupe Hospitalier Pitié Salpêtrière, 75013 Paris, France

4 Department of Neurosurgery, AP-HP, Groupe Hospitalier Pitié Salpêtrière, 75013 Paris, France removable, to highly aggressive grade III recurrent metastatic lesions: patients need iterative operations in 38-51\% of cases and will develop systemic metastases in 10-20\% $[3,4]$. Even though radiation therapy improves progressionfree survival, there is no effective treatment for patients with multiple meningeal lesions not amenable to surgery.

The NAB2-STAT6 fusion protein drives SFT/H tumorigenesis by converting the early growth response (EGR) repressor NGFI-A Binding 2 (NAB2) into an EGR activator. While there is currently no direct inhibitor of NAB2 and EGR, the inhibition of downstream EGR targets could be considered for treatment of SFT/H: indeed, the NAB2-STAT6 fusion activates a wide panel of EGR1 downstream targets, including Fibroblast Growth Factor Receptor 1 (FGFR1), NTRK1, IGF2 [1, 5]. However, until now, no pharmacological treatment, including Bevacizumab and Sunitinib, provides more than a transient stabilization (Stable Disease according to RECIST or RANO) in CNS SFT/H [6]. Pazopanib, usually proposed in renal cell carcinoma, and recommended in soft tissue sarcomas [7], acts as a broad-spectrum tyrosine kinase inhibitor and targets FGFR1 and PlateletDerived Growth Factor Receptor (PDGFR) more efficiently 
than Sunitinib [8]. We therefore treated with Pazopanib two patients with malignant SFT/H recurring after iterative surgery and radiotherapy. In both cases, a rapid and spectacular regression was observed.

The first patient is a 31 year-old male with a history of recurrent CNS SFT/H for 11 years. Despite three operations (grade I SFT/H confirmed by positive STAT6 nuclear staining at first surgery, then grade III) and five sessions of stereotactic radiosurgery, multiple tumor nodules were rapidly progressing involving the occipital regions, parietal convexity, tentorium, and along the spinal cord. The patient had recent neurological deterioration with cerebellar syndrome, vertigo, nausea, headaches and a Karnofsky performance status (KPS) of 70. There was no systemic symptomatic metastasis.

The patient was enrolled in the whole exome sequencing and RNAseq program EXORARE. A fusion between NAB2exon6 and STAT6exon17 genes was identified, a classical fusion type in CNS SFT/H [1], but no other relevant alteration was found, with an overall low mutation frequency. RNAseq of this sample in addition to another meningeal SFT/H (GSE66729 [9], MCTP_SAMPLE_232) and compared to the transcriptomic profiling of 5 systemic SFT/H (GSE42948 [10], samples STT2162_SFT to STT6077_SFT) revealed a significant (false discovery rate, FDR, p-value $<0.05$ ) upregulation of EGR dependent targets PDGFRa, FGFR1 and downregulation of PDGFRb (cf. Supplementary Table 1). The pathway analysis pinpointed a broad range overexpression of the different tyrosine kinases targeted by pazopanib (cf. Fig. 1). Further details on the methods of differential expression analysis of the different RNAseq datasets are provided as Supplementary Methods. Based on these data, we started oral Pazopanib $800 \mathrm{mg}$ daily, which was decreased after 1 month to $600 \mathrm{mg}$ because of digestive side effects. After 2 months, neurological status improved and the multiple nodules of the tumor dramatically decreased on MRI, with a stable control at 4 months. For instance, the three main posterior fossa lesions decreased from a total volume of 11.6 to $1.9 \mathrm{~cm}^{3}$ (84\% decrease) (cf. Fig. 2a, b).

Second patient is a 52 year-old woman diagnosed with a right petrous temporal grade II SFT/H at 29 years. In 23 years, she underwent four neurosurgical resections, three sessions of stereotactic radiosurgery and a course of hypofractioned radiotherapy. On neurological examination, she had partial facial palsy and a KPS of 90 . The meningeal lesion rapidly grew within 8 months, with the development of subcutaneous bulking. Molecular analysis was not performed in this patient but nuclear STAT6 immunostaining was positive, which is used as a standard diagnosis tool in $\mathrm{SFT} / \mathrm{H}$ and indirectly proves that a NAB2-STAT6 fusion has occurred [1]. Therefore, we administrated to the patient oral Pazopanib 600 mg daily, which was well tolerated.

\section{Enriched Terms}

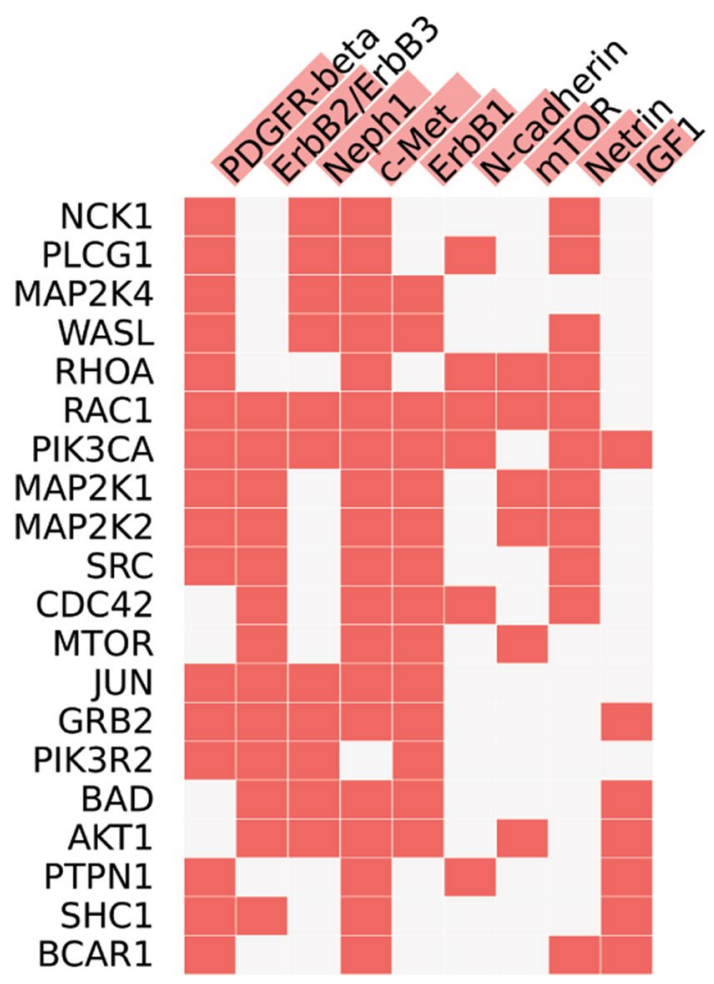

Fig. 1 The ten most significant enriched pathway signaling using Enrichr and NCI Nature Pathway Database and considering the most differentially expressed genes (absolute $\log 2$ fold-change $>2$ with a False discovery-rate p-value $<0.0005)$. Columns represent the signaling pathways and rows the 20 more frequently represented genes within these pathways, the red boxes indicate that a gene belong to a specific pathway signaling

The patient noticed a rapid regression of the subcutaneous bulk and after 6 months the tumor decreased from 85.9 to $49.0 \mathrm{~cm}^{3}$ (43\% decrease) (cf. Fig. 2c, d).

Most of the gene fusions recurrently found in human cancers produce highly oncogenic proteins and are master regulators of tumorigenesis process [11]. They represent therefore ideal candidates for target therapies [12]. In the case of SFT/H, NAB2-STAT6 is found in $100 \%$ of the tumors $[1,2]$ and high throughput gene sequencing performed in our first patient failed to identify any other relevant oncogenic event, comforting the hypothesis that NAB2-STAT6 is the main driver of SFT/H growth. Several treatments have been tested that could affect the EGR downstream effectors, such as VEGF-A with the Bevacizumab or PDGFRa with the Sunitib [6]. However, in contrast with the dramatic responses we observed in our two cases, Pazopanib showed a modest efficacy in SFT/H subcutaneous xenografts [6] and in patients with systemic SFT/H, leading to tumor stabilization with only one partial response [6, 13-16]. Only one reported case had a CNS SFT/H but the treatment was 
Fig. 2 a, b MRI evolution of a grade III meningeal SFT in patient 1. a Before Pazopanib. Axial and sagittal T1-weighted contrast-enhanced brain MRI show multiple nodules disseminated in the supra-tentorial and infra-tentorial spaces, the basal cisterns and along the ventricles. b Four months after the introduction of oral Pazopanib. Most nodules have disappeared and the main ones have decreased. For instance, the three main posterior fossa lesions decreased from a total volume of 11.6 to $1.9 \mathrm{~cm}^{3}$ ( $84 \%$ decrease). c, d MRI evolution of a grade II meningeal SFT in patient 2. c In 8 months, the tumor recurred rapidly, disseminating to the petrous bone, temporal lobe and subcutaneous tissues, without neurological worsening. d Six months after the introduction of oral Pazopanib. The mass has decreased from 85.9 to $49.0 \mathrm{~cm}^{3}(43 \%$ decrease) and the temporal bulk was not clinically noticeable anymore

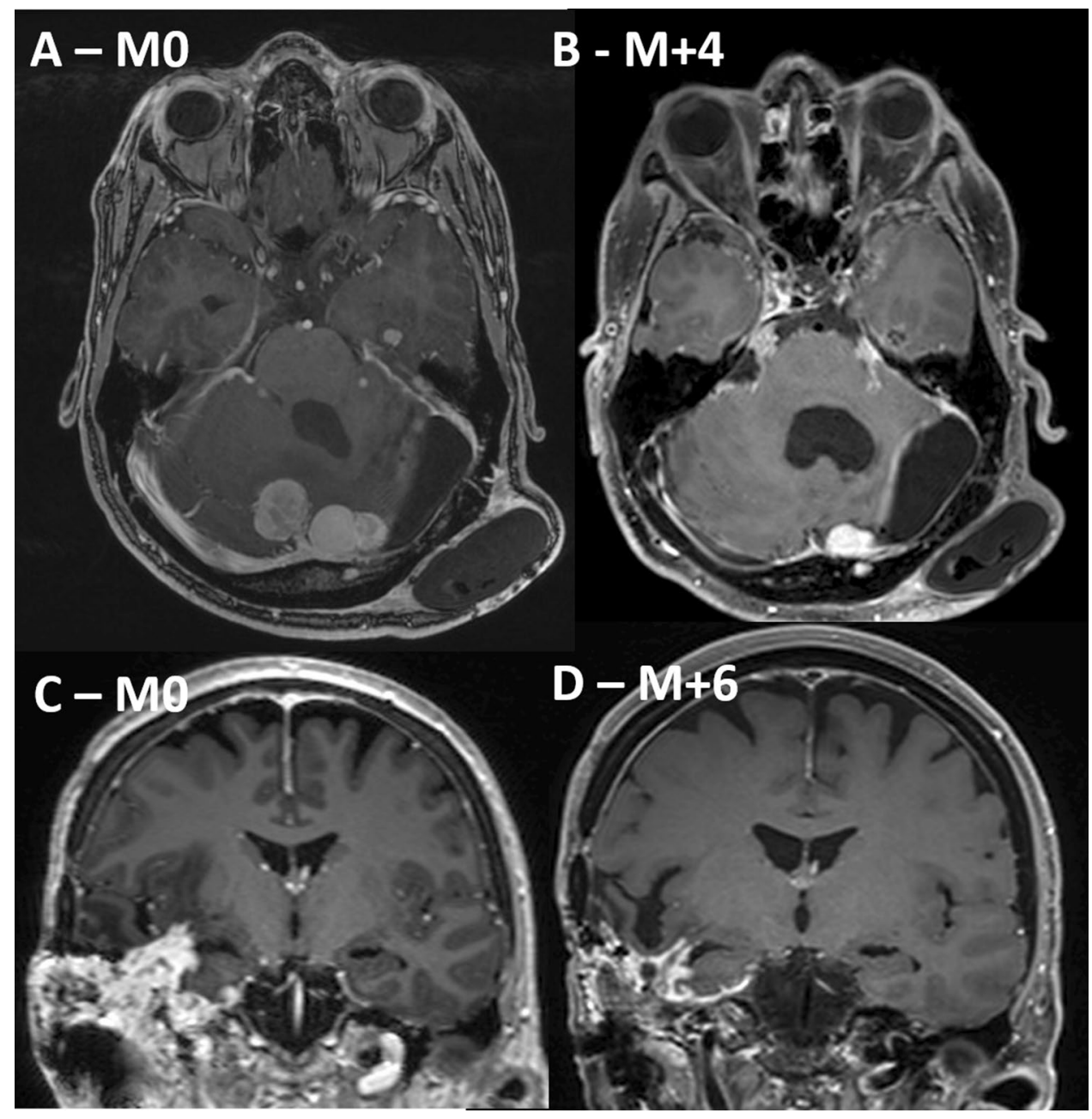

stopped after 10 days and the efficacy could not be evaluated [7]. This difference in Pazopanib response may be related to the significantly different expression profile found in the two analyzed meningeal SFT/H (our case 1 and MCTP_SAMPLE_232) as compared to five systemic SFT transcriptomic profiles (cf. Supplementary Table 1). Accordingly, the pathway analysis of the most significantly differential expressed genes, also pinpointed an enrichment of several tyrosine kinase signaling targeted by Pazopanib (cf. Fig. 1). An ongoing phase II open-label, non-randomized trial proposes Pazopanib to patients with unresectable or metastatic SFT (NCT02066285), but it excludes meningeal lesions. Our results suggest that Pazopanib has a higher efficacy in the CNS SFT/H compared to systemic SFT/H and strongly support a more extensive study.

Acknowledgement We are indebted to Pr P Laurent-Puig for NGS analysis. This work was supported by the grant EXORARE from the Cancéropôle Ile de France/INCa.

Author contributions Conception and design: MK, MS. Acquisition of data: KM, MK, MS. Analysis and interpretation of data: CA, AA,
KM, MK, MS. Drafting the article: CA. Critically revising the article: AA, MK, MS. Approved the final version of the manuscript: CA, AA, KM, MK, MS. Supervision: MS.

\section{Compliance with ethical standards}

Conflict of interest The authors declare no conflict of interest.

Informed consent The patients gave their written consent.

\section{References}

1. Robinson DR, Wu Y-M, Kalyana-Sundaram $\mathrm{S}$ et al (2013) Identification of recurrent NAB2-STAT6 gene fusions in solitary fibrous tumor by integrative sequencing. Nat Genet 45(2):180-185

2. Chmielecki J, Crago AM, Rosenberg M et al (2013) Whole-exome sequencing identifies a recurrent NAB2-STAT6 fusion in solitary fibrous tumors. Nat Genet 45(2):131-132

3. Apra C, Mokhtari K, Cornu P et al (2017) Intracranial solitary fibrous tumors/hemangiopericytomas: first report of malignant progression. J Neurosurg. 2017:1-6

4. Champeaux C, Khan AA, Wilson E et al (2017) Meningeal haemangiopericytoma and solitary fibrous tumour: a retrospective 
bi centre study for outcome and prognostic factor assessment. J Neurooncol 134:387-395

5. Park MS, Ravi V, Araujo DM (2010) Inhibiting the VEGFVEGFR pathway in angiosarcoma, epithelioid hemangioendothelioma, and hemangiopericytoma/solitary fibrous tumor. Curr Opin Oncol 22(4):351-355

6. Stacchiotti S, Tortoreto M, Baldi GG et al (2014) Preclinical and clinical evidence of activity of pazopanib in solitary fibrous tumour. Eur J Cancer 50(17):3021-3028

7. Chellappan DK, Chellian J, Ng ZY et al (2017) The role of pazopanib on tumour angiogenesis and in the management of cancers: a review. Biomed Pharmacother 96:768-781

8. Schutz FAB, Choueiri TK, Sternberg CN (2011) Pazopanib: clinical development of a potent anti-angiogenic drug. Crit Rev Oncol/ Hematol 77(3):163-171

9. Balbin OA, Malik R, Dhanasekaran SM et al (2015) The landscape of antisense gene expression in human cancers. Genome Res 25(7):1068-1079

10. Guo X, Zhu SX, Brunner AL et al (2013) Next generation sequencing-based expression profiling identifies signatures from benign stromal proliferations that define stromal components of breast cancer. Breast Cancer Res 15(6):R117

11. Project ICGCPT, Bender S, Gronych J et al (2016) Recurrent MET fusion genes represent a drug target in pediatric glioblastoma. Nat Med 22(11):1314-1320
12. Cools J, DeAngelo DJ, Gotlib J et al (2003) A tyrosine kinase created by fusion of the PDGFRA and FIP1L1 genes as a therapeutic target of imatinib in idiopathic hypereosinophilic syndrome. $\mathrm{N}$ Engl J Med 348(13):1201-1214

13. Kasper B, Sleijfer $S$, Litière $S$ et al (2014) Long-term responders and survivors on pazopanib for advanced soft tissue sarcomas: subanalysis of two European Organisation for Research and Treatment of Cancer (EORTC) clinical trials 62043 and 62072. Ann Oncol 25(3):719

14. Levard A, Derbel O, Méeus P et al (2013) Outcome of patients with advanced solitary fibrous tumors: the Centre Léon Bérard experience. BMC Cancer 13:109

15. Maruzzo M, Martin-Liberal J, Messiou C et al (2015) Pazopanib as first line treatment for solitary fibrous tumours: the Royal Marsden Hospital experience. Clin Sarcoma Res 5(1):5

16. Lee SJ, Kim ST, Park SH et al (2014) Successful use of pazopanib for treatment of refractory metastatic hemangiopericytoma. Clin Sarcoma Res 4:13

17. Benjamini Y, Hochberg Y (1995) Controlling the false discovery rate: a practical and powerful approach to multiple testing. J R Stat Soc Ser B (Methodological) 57(1):289-300 